\title{
Molecular epidemiology and genotype-phenotype correlation in phenylketonuria patients from South Spain
}

\author{
María A Bueno ${ }^{1}$, Domingo González-Lamuño ${ }^{2}$, Carmen Delgado-Pecellín ${ }^{3}$, Luís Aldámiz-Echevarría ${ }^{4}$, \\ Belén Pérez ${ }^{5}$, Lourdes R Desviat ${ }^{5}$ and María L Couce ${ }^{6}$
}

The aim of this study was to identify the most common genotypes in the phenylketonuria (PKU) population of Andalusia, assessing the correlation with the phenotype and the usefulness in predicting the response to treatment with tetrahydrobiopterin. We conducted a retrospective observational study between January 1980 and January 2010 in 147 Andalusian PKU patients assessing phenotype, genotype and response to a 24-h BH4 loading test. Our cohort of patients exhibited 65 different mutations, $69.2 \%$ corresponding to the missense type, in a total of 123 different genotypes. IVS10nt$11 \mathrm{~g}>\mathrm{a}$ was the most common mutation (10.9\%). Four novel missense mutations were identified: p.L258P; p.E66K, p.R155C and p.P122S. Although generally there is a good genotype-phenotype correlation, for eight of the repeated genotypes a slightly different phenotype was observed. In 96 PKU subjects BH4 challenge was carried out. Patients with previously reported unresponsive mutations on both alleles showed a negative response, while $95.5 \%(28 / 29)$ of the responsive patients carry at least one missense mutation previously associated to the BH4. Our data reveal a great genetic heterogeneity in the Andalusian population. Genotype is quite a good predictor of the phenotype and of the responsiveness to tetrahydrobiopterin, which is relevant for patient management and follow-up.

Journal of Human Genetics (2013) 58, 279-284; doi:10.1038/jhg.2013.16; published online 21 March 2013

Keywords: genotype-phenotype correlation; hyperphenylalaninemia; missense mutations; phenylketonuria; rare disease; tetrahydrobiopterin

\section{INTRODUCTION}

Phenylketonuria (PKU, OMIM 261600) is the most common inborn error of amino-acid metabolism in Caucasians, where 1 in 10000 individuals is affected (carriers 1/50). ${ }^{1}$ The incidence of the disorder in Andalusia population (South of Spain) is 1 in 12000 with a carrier frequency of 1 in $55 .^{2}$ It is caused by alterations of the $P A H$ gene coding for L-phenylalanine-4-hydroxylase (PAH, EC1.14.16.1). The $\mathrm{PAH}$ locus includes 13 exons and spans $90 \mathrm{~kb}$. There are more than 700 different allelic variants known to affect enzymatic activity at this locus. Every exon, and most introns, has at least one known mutation (http://www.pahdb.mcgill.ca). About one-third of the mutations produce non-clinical elevation of blood phenylalanine levels (benign hyperphenylalaninemia (HPA)). Among the remaining two-thirds that lead to PKU, more than half are missense mutations leading to amino-acid substitutions and cause reduced or no enzyme function by a variety of pathogenetic mechanisms. Other types of mutations include nonsense mutations, small insertion or deletions, splicing defects. Large deletions have also been recently described. ${ }^{3-5}$

A broad spectrum of the PAH deficiency phenotypes can be found: benign HPA, mild PKU, moderate PKU and classical PKU. A subset of the patients exhibit cofactor (BH4) responsiveness depending on the genotype. ${ }^{6,7}$ Genotype-phenotype correlations have been extensively analyzed revealing they can be a strong and reliable predictive tool. ${ }^{7,8-10}$ Accordingly, depending on the specific genotype, a better-tailored diet and the potential BH4 responsiveness can be established. Almost 1200 genotypes associated with $\mathrm{BH} 4$ responsiveness are described and listed in the BIOPKU database (www.biopku.org/biopku). As extensively shown by expression analysis in different systems, a decrease in protein stability associated to missense mutations is the main molecular pathogenic mechanism in PKU and the determinant for phenotypic outcome and $\mathrm{BH} 4$ responsiveness, which is mainly due to a stabilizing chaperone effect of the cofactor. ${ }^{11,12}$

\footnotetext{
${ }^{1}$ Unit of Metabolophaties and Nutrition, Department of Pediatrics, Hospital Universitario Virgen del Rocio, Sevilla, Spain; ${ }^{2}$ Paediatric Nephrology and Metabolism Unit, Department of Pediatrics, Hospital Marqués de Valdecilla, Santander, Spain; ${ }^{3}$ Department of Clinical Biochemical, Hospital Universitario Virgen del Rocío, Sevilla, Spain; ${ }^{4}$ Division of Metabolism, Department of Pediatrics, Hospital de Cruces, Vizcaya, Spain; ${ }^{5}$ Centro de Diagnóstico de Enfermedades Moleculares, Centro de Biología Molecular Severo Ochoa, CSIC-UAM, CIBERER, IdiPaz, Madrid, Spain and ${ }^{6}$ Unit of Diagnosis and Treatment of Congenital Metabolic Diseases, Department of Pediatrics, Hospital Clinico Universitario de Santiago, A Coruña, Spain

Correspondence: Dr ML Couce, Unit of Diagnosis and Treatment of Congenital Metabolic Diseases, Department of Pediatrics, Hospital Clinico Universitario de Santiago, Travesía da Choupana s/n, Santiago de Compostela, A Coruña 15706, Spain.

E-mail: maria.luz.couce.pico@sergas.es
}

Received 20 November 2012; revised 7 February 2013; accepted 17 February 2013; published online 21 March 2013 
Although residual activity for each mutation has been established in vitro, there are situations without coincidence between the residual in vitro activity and the clinical phenotype of the patient. Even brothers with identical genotype can exhibit different clinical phenotypes. ${ }^{13,14}$ In addition, about $75-85 \%$ of the PKU patients are compound heterozygotes ${ }^{15}$ and at the end clinical and biochemical phenotypes depend on the interaction between the proteins produced by both alleles. Differences in the responsiveness to $\mathrm{BH} 4$ and in the correlation between genotype-phenotype could also be partially due to differences in the management, clinical criteria and environment.

A marked genetic heterogeneity in PAH mutations has been observed in different populations. Although the information in PAHdb (for $\sim 450$ variant alleles, carried on $>8000$ independent human chromosomes) is already quite extensive, some populationspecific information is still missing on the web site (http:// www.pahdb.mcgill.ca). The p.R408W mutation is the most prevalent mutation worldwide, whereas its allele frequency varies considerably within Europe, being $<5 \%$ in Southern Europe, ${ }^{16-18}$ while IVS10$11 \mathrm{G}>\mathrm{A} \quad(\mathrm{c} .1066-11 \mathrm{~g}>\mathrm{a})$ mutation is the major PKU-causing mutation throughout the Mediterranean region. ${ }^{18-20}$ In the oriental population a different mutational profile is observed. ${ }^{21}$ In Spain there are also significant regional differences in the mutational spectrum, reflecting the genetic ancestry of each geographical region. ${ }^{17}$
The approach from the clinical and biochemical parameters to the gene mutations should improve our knowledge about the $P A H$ gene, its alleles and their significance. Descriptions from areas with genetic diversity but homogeneous clinical approach seem to be relevant. In this report we present the mutational spectrum of PAH patients in the Andalusian population compared with the rest of Spain and other countries in Europe. We describe the genotype-phenotype relationship and evaluate the response to $\mathrm{BH} 4$ to expand the knowledge of the molecular epidemiology of the disease and the BH4-responsive genotypes.

\section{MATERIALS AND METHODS}

\section{Subjects}

Patients were evaluated for diagnosis and treatment at the Congenital Metabolic Disease Unit, Hospital Virgen del Rocío of Sevilla, between January 1980 and January 2010. This unit controlled Andalusian patients during that period as a reference center for this disease. Andalusia, region to the South of Spain, has 10000000 inhabitants and represents $22 \%$ of the Spanish population. We detected in this period 179 patients ( 78 male and 91 female) of the Andalusian community whose cut-off value for phenylalanine is $180 \mu \mathrm{m}$. Most were studied as part of the PKU screening program in the neonatal period, launched in the year 1979 in Seville, but some were studied also after presenting psychomotor delay or because they had a relative diagnosed with HPA.

Table 1 Mutational spectrum in the Andalusian PKU population, indicating the number of alleles for each mutation and their relative residual PAH activity in vitro according to PAHdb (http://www.pahdb.mcgill.ca)

\begin{tabular}{|c|c|c|c|c|c|c|c|}
\hline PAH mutation & Nucleotide & Activity \% & No. of alleles & PAH mutation & Nucleotide & Activity \% & No. of alleles \\
\hline IVS10-11g>a & c. $1066-11 \mathrm{~g}->\mathrm{a}$ & 0 & 32 & p.G289R & c. $865 \mathrm{G}>\mathrm{C}$ & $?$ & 2 \\
\hline p.S349P & c. $1045 \mathrm{~T}>\mathrm{C}$ & 1 & 24 & p.G352fs & c. 1055 delG & Null & 2 \\
\hline p.V388M & c. $1162 \mathrm{G}>\mathrm{A}$ & 28 & 22 & p.G46S & c. $136 \mathrm{G}>\mathrm{A}$ & 16 & 2 \\
\hline IVS4 $+5 g>t$ & c. $441+5 g>t$ & Null & 17 & p.L258P & c.773T $>C$ & $?$ & 2 \\
\hline p.l65T & c. $194 \mathrm{~T}>\mathrm{C}$ & 33 & 13 & p.L348V & c. $1042 C>G$ & 35 & 2 \\
\hline p.R261P & c. $782 \mathrm{G}>\mathrm{C}$ & 32 & 13 & p.R241H & c. $722 \mathrm{G}>\mathrm{A}$ & 23 & 2 \\
\hline p.R261Q & c. $782 \mathrm{G}>\mathrm{A}$ & 44 & 13 & p.R243X & c. $727 \mathrm{C}>\mathrm{T}$ & 0 & 2 \\
\hline p.A403V & c. $1208 \mathrm{C}>\mathrm{T}$ & 66 & 10 & p.S87R & c. $261 \mathrm{C}>\mathrm{A}$ & 82 & 2 \\
\hline IVS12 $+1 \mathrm{~g}>\mathrm{a}$ & c. $1315+1 \mathrm{G}>\mathrm{A}$ & 0 & 9 & p.A104D & c. $311 \mathrm{C}>\mathrm{A}$ & 27 & 1 \\
\hline p.R68S & c. $204 \mathrm{~A}>\mathrm{T}$ & 98 & 9 & p.D145N & c. $433 \mathrm{G}>\mathrm{A}$ & $?$ & 1 \\
\hline p.Y414C & c. $1241 A>G$ & 57 & 8 & p.D145V & c. $434 \mathrm{~A}>\mathrm{T}$ & $?$ & 1 \\
\hline p.R408W & c. $1222 \mathrm{C}>\mathrm{T}$ & 2 & 7 & p.E66K & c. $196 \mathrm{G}>\mathrm{A}$ & $?$ & 1 \\
\hline p.Q304Q & c. $912 \mathrm{G}>\mathrm{A}$ & Null & 6 & p.F39L & c. $117 \mathrm{C}>\mathrm{G}$ & 50 & 1 \\
\hline p.E390G & c. $1169 A>G$ & 62 & 5 & p.F55fs & c.165delT & Null & 1 \\
\hline p.R158Q & c. $473 \mathrm{G}>\mathrm{A}$ & 10 & 5 & p.1421T & c. $1262 \mathrm{~T}>\mathrm{C}$ & $?$ & 1 \\
\hline p.R243Q & c. $728 \mathrm{G}>\mathrm{A}$ & 13 & 5 & IVS2 $+5 g>a$ & c. $168+5 g->a$ & Null & 1 \\
\hline p.A300s & c. $898 \mathrm{G}>\mathrm{T}$ & 31 & 4 & IVS4-5c >g & c. $442-5 c->g$. & Null & 1 \\
\hline p.F55L & c. $165 \mathrm{~T}>\mathrm{G}$ & $?$ & 4 & p.N61K & c. $183 C>G$ & $?$ & 1 \\
\hline IVS1 $+5 g>t$ & c. $60+5 G>T$ & Null & 4 & p.N438fs & c. $1314-1315+4$ del 6 & $?$ & 1 \\
\hline IVS8nt-7a >g & 913-7a->g. & Null & 4 & p.P122S & c. $364 \mathrm{C}>\mathrm{T}$ & $?$ & 1 \\
\hline p.Y204X & c. $612 \mathrm{~T}>\mathrm{G}$ & 0 & 4 & p.P275R & c. $824 \mathrm{C}>\mathrm{G}$ & $?$ & 1 \\
\hline p.E280K & c. $838 \mathrm{G}>\mathrm{A}$ & 0 & 3 & p.Q20X & c. $58 \mathrm{C}>\mathrm{T}$ & $?$ & 1 \\
\hline p.G272X & c. $814 \mathrm{G}>\mathrm{T}$ & 0 & 3 & p.R155C & c. $463 \mathrm{C}>\mathrm{T}$ & $?$ & 1 \\
\hline p.M1I & c. $3 \mathrm{G}>\mathrm{A}$ & $?$ & 3 & p.R176L & c. $527 \mathrm{G}>\mathrm{T}$ & 42 & 1 \\
\hline p.P281L & c. $842 \mathrm{C}>\mathrm{T}$ & 2 & 3 & p.R176X & c. $526 \mathrm{C}>\mathrm{T}$ & Null & 1 \\
\hline p.P362T & c. $1084 C>A$ & $?$ & 3 & p.R261X & c. $781 \mathrm{C}>\mathrm{T}$ & Null & 1 \\
\hline p.R252Q & c. $755 \mathrm{G}>\mathrm{A}$ & 3 & 3 & p.R265Q & c. $782 \mathrm{G}>\mathrm{A}$ & $?$ & 1 \\
\hline p.A309V & c. $926 \mathrm{C}>\mathrm{T}$ & 70 & 2 & p.T380M & c. $1139 \mathrm{C}>\mathrm{T}$ & $?$ & 1 \\
\hline p.A47V & c. $140 \mathrm{C}>\mathrm{T}$ & $16-40$ & 2 & p.V230I & c. $688 \mathrm{G}>\mathrm{A}$ & $50-65$ & 1 \\
\hline p.C217G & c. $649 \mathrm{~T}>\mathrm{G}$ & $?$ & 2 & p.V245A & c.734T $>C$ & 63 & 1 \\
\hline p.D415N & c. $1243 \mathrm{G}>\mathrm{A}$ & $72-100$ & 2 & p.Y198fs & c.593_614 del22 & 1 & 1 \\
\hline del F39 & c.115-117 delTTC & 20 & 2 & p.Y206X & c. $618 \mathrm{C}>\mathrm{G}$ & Null & 1 \\
\hline p.E178G & c. $533 A>G$ & 39 & 2 & Unknown & & & 6 \\
\hline
\end{tabular}

Abbreviations: null, predicted null activity; PAH, phenylalanine-4-hydroxylase; PKU, phenylketonuria; ?, unknown. 
Overall, we studied 147 patients (among them seven pairs of siblings). Patients with transient HPA (two patients) or with HPA due to a defect in the synthesis or recycling of the cofactor $\mathrm{BH} 4$ (two patients) were excluded. Blood phenylalanine concentrations at diagnosis and/or dietary tolerance at 5 years of age and genotype were assessed in all the patients, and their answer to $\mathrm{BH} 4$ loading test was assessed in 96 of them.

This study was approved by the local Ethics Committee, and informed consents were obtained from the patients or from their parents.

\section{Type of HPA}

Patients were classified according to their levels of phenylalanine at diagnosis and/or dietary tolerance at 5 years old in classic PKU (Phe $\geqslant 1200 \mu \mathrm{M}$, tolerance $\leqslant 350 \mathrm{mg}$ per day), moderate PKU (Phe between $600-1200 \mu \mathrm{m}$, tolerance 350 $600 \mathrm{mg}$ per day), mild PKU (Phe between $300-600 \mu \mathrm{M}$, tolerance $600-800 \mathrm{mg}$ per day) and benign HPA (phe $<300 \mu \mathrm{m}$ on a normal diet).

\section{Genotype analysis}

Genomic DNA of patients and their families was obtained from whole blood samples. Genetic analyses were performed at the Centro de Diagnóstico de Enfermedades Moleculares, Universidad Autónoma de Madrid, by a combined approach using Denaturing Gradient Gel Electrophoresis and direct sequencing using the BigDye Terminator v.3.1 (Applied Biosystems, Foster City, CA, USA) kit and capillary electrophoresis with a genetic Analyzer ABI Prism 3700 (Applied Biosystems). Patient samples where only one causative mutation was identified were subjected to MLPA analysis (SALSA MLPA P055 PAH Probemix, MRC-Holland, Amsterdam, The Netherlands) to exclude large deletions/duplications.

PAH residual activity for each mutant protein was assessed from data compiled in the PAHdb (www.pahdb.mcgill.ca) and/or BH4db (www.bh4.org).

\section{BH4 loading test}

The BH4 loading tests considered in this study were performed between 2005 and 2009. The initial stage of the protocol considered a reduction of at least $50 \%$ in blood Phe $24 \mathrm{~h}$ after a load of L-Phe $\left(100 \mathrm{mg} \mathrm{kg}^{-1}\right.$; Nutricia S.R.L., Madrid, Spain) and subsequent intake of (6R)-BH4 $\left(20 \mathrm{mg} \mathrm{kg}^{-1}\right.$; Schicks Laboratories, Jona, Switzerland) $3 \mathrm{~h}$ later as the criterion for considering an individual to be $\mathrm{BH} 4$ responsive. ${ }^{22}$ PKU patients who did not meet the aforementioned criterion for responsiveness subsequently underwent the therapeutic test to confirm whether non-responders in the 24-h test were indeed non-responders to $\mathrm{BH} 4$ treatment. This second stage of the protocol entails administering a BH4 dose of $20 \mathrm{mg} \mathrm{kg}^{-1}$ per day for 1 week and a daily protein intake meeting patients' age- and sex-specific RDAs. A Phe level, at the end of this period, remaining below a defined threshold $\left(<360 \mu \mathrm{moll}^{-1}\right.$ for individuals $<6$ years of age; $<480 \mu \mathrm{moll}^{-1}$ for those 6 to $<10$ years of age, and $<600 \mu \mathrm{moll}^{-1}$ for those $>10$ years of age) was considered a positive result in the therapeutic test.

Prediction of BH4 responsiveness according to the genotype was established (www.bh4.org and www.pahdb.mcgill.ca).

\section{RESULTS}

According to the phenotype, $32(21.8 \%)$ of the 147 patients were classified as benign HPA, $18(12.2 \%)$ as mild PKU, $38(25.8 \%)$ as moderate PKU and $59(40.1 \%)$ as classic PKU. Genetic analysis resulted in the identification of two deleterious mutations in all patients but six in whom only one causative mutation was identified after sequencing all exons and excluding large deletions by MLPA analysis.

The mutational spectrum includes 65 distinct mutations, 45 $(69.2 \%)$ corresponding to different missense mutations leading to amino-acid substitutions, 9 (13.8\%) splicing errors, $6(9.2) \%$ are nonsense mutations and $5(7.7 \%)$ are small deletions (Table 1). Mutations were identified in all exons except number 13; exon 7 exhibits the greatest number of different mutations (15) and is affected in $15.9 \%$ of the total mutant alleles.
Among the 294 alleles analyzed, only the common Mediterranean mutation, IVS1011 $\mathrm{g}>\mathrm{a}(\mathrm{c} .1066-11 \mathrm{~g}>\mathrm{a})$, displayed a relative high frequency of $10.9 \%$. The sum of another seven mutations accounts for $38.1 \%$ of all mutated alleles (in order of frequency p.S349P, p.V388M, IVS4 $+5 g>t \quad(c .441+5 g>t), \quad$ p.I65T, p.R261Q, p.R261P and p.A403V) (Table 1). Four of the mutations identified in our population are novel changes not present in $>5000$ individuals sequenced (Exome Variant Server: http://evs.gs.washington.edu/EVS/): p.P122S (c.364C > T), p.L258P (c.773T >C), p.E66K (c.196G >A) and p.R155C (c.463C $>$ T). Combination of all the mutations generates up to 123 different genotypes in the 147 patients. Just 12 genotypes are present more than once in unrelated patients (and seven genotypes repeated in siblings) (Table 2). The majority of patients display compound heterozygosis for two different mutations, and only 24 $(16.3 \%)$ are homozygous. Genetic analysis in parents confirmed that the patients are homozygous. Among these, 25\% (6 cases) present the IVS10-11g $>$ a mutation, 5 patients are homozygous for p.S349P, 3 patients for IVS12 $+1 \mathrm{~g}>\mathrm{a}$ and 2 patients for p.R408W.

Regarding the enzymatic activity associated to each mutation, thus determinant of the theoretical clinical phenotype of each patient, $41.5 \%$ are associated with a null to very low PAH activity $(<10-15 \%$ in vitro enzyme activity), $20 \%$ with a moderate enzymatic activity (15-30\% in vitro enzyme activity) and 30.9\% with a high residual activity ( $>30 \%$ residual activity) (Table 1 ). Among homozygous patients, those carrying severe or null mutations (IVS10-11g $>a$, p.S349P, IVS12 + 1g > a, p.R408W, p.P281L) were classified as classic PKU. However, although a good genotype-phenotype correlation is

Table 2 Genotypes present in more than one patient and their corresponding phenotype

\begin{tabular}{|c|c|c|c|}
\hline Allele 1 & Allele 2 & Phenotype & Relationship \\
\hline p.A403V & p.V388M & $2 \mathrm{HPA}$ & Siblings \\
\hline p.A403V & p.R261Q & $\begin{array}{l}1 \text { MPKU } \\
1 \text { BHPA }\end{array}$ & No \\
\hline p.165T & p.Q304Q & $2 \mathrm{CPKU}$ & No \\
\hline IVS10-11g >a & IVS10-11g >a & $\begin{array}{l}4 \mathrm{CPKU} \\
2 \mathrm{MPKU}\end{array}$ & No \\
\hline IVS12 $+1 \mathrm{~g}>\mathrm{a}$ & IVS12 $+1 g>a$ & $3 \mathrm{CPKU}$ & No \\
\hline $\operatorname{IVS} 1+5 g>t$ & p.R261Q & $\begin{array}{l}1 \mathrm{CPKU} \\
1 \mathrm{MPKU}\end{array}$ & Siblings \\
\hline IVS4 $+5 g>t$ & IVS10-11g >a & $2 \mathrm{MPKU}$ & No \\
\hline IVS4 $+5 g>t$ & p.V388M & $\begin{array}{l}1 \mathrm{CPKU} \\
4 \mathrm{MPKU}\end{array}$ & No \\
\hline p.M1I & F55L & $\begin{array}{l}1 \mathrm{mPKU} \\
1 \mathrm{BHPA}\end{array}$ & Siblings \\
\hline p.R243Q & IVS10-11g >a & $\begin{array}{l}1 \mathrm{CPKU} \\
1 \mathrm{MPKU}\end{array}$ & No \\
\hline p.R261P & p.165T & $2 \mathrm{CPKU}$ & Siblings \\
\hline p.R261Q & p.R261P & $3 \mathrm{MPKU}$ & No \\
\hline p.R408W & p.R408w & $2 \mathrm{CPKU}$ & No \\
\hline p.S349P & p.R261Q & $2 \mathrm{MPKU}$ & No \\
\hline p.S349P & p.S349P & $5 \mathrm{MPKU}$ & No \\
\hline p.S349P & p.V388M & $2 \mathrm{CPKU}$ & No \\
\hline p.V388M & IVS8-7a>g & $\begin{array}{l}2 \mathrm{CPKU} \\
1 \mathrm{MPKU}\end{array}$ & Siblings \\
\hline p.Y204X & p.R68S & $\begin{array}{l}2 \mathrm{CPKU} \\
2 \mathrm{MPKU}\end{array}$ & Siblings \\
\hline p.Y414C & p.G46S & $2 \mathrm{MPKU}$ & Siblings \\
\hline
\end{tabular}

Abbreviations: BHPA, benign hyperphenylalaninemia; CPKU, classical phenylketonuria; HPA, hyperphenylalaninemia; mPKU, mild phenylketonuria; MPKU, moderate phenylketonuria. 
Table 3 Response to the 24-h BH4 loading test

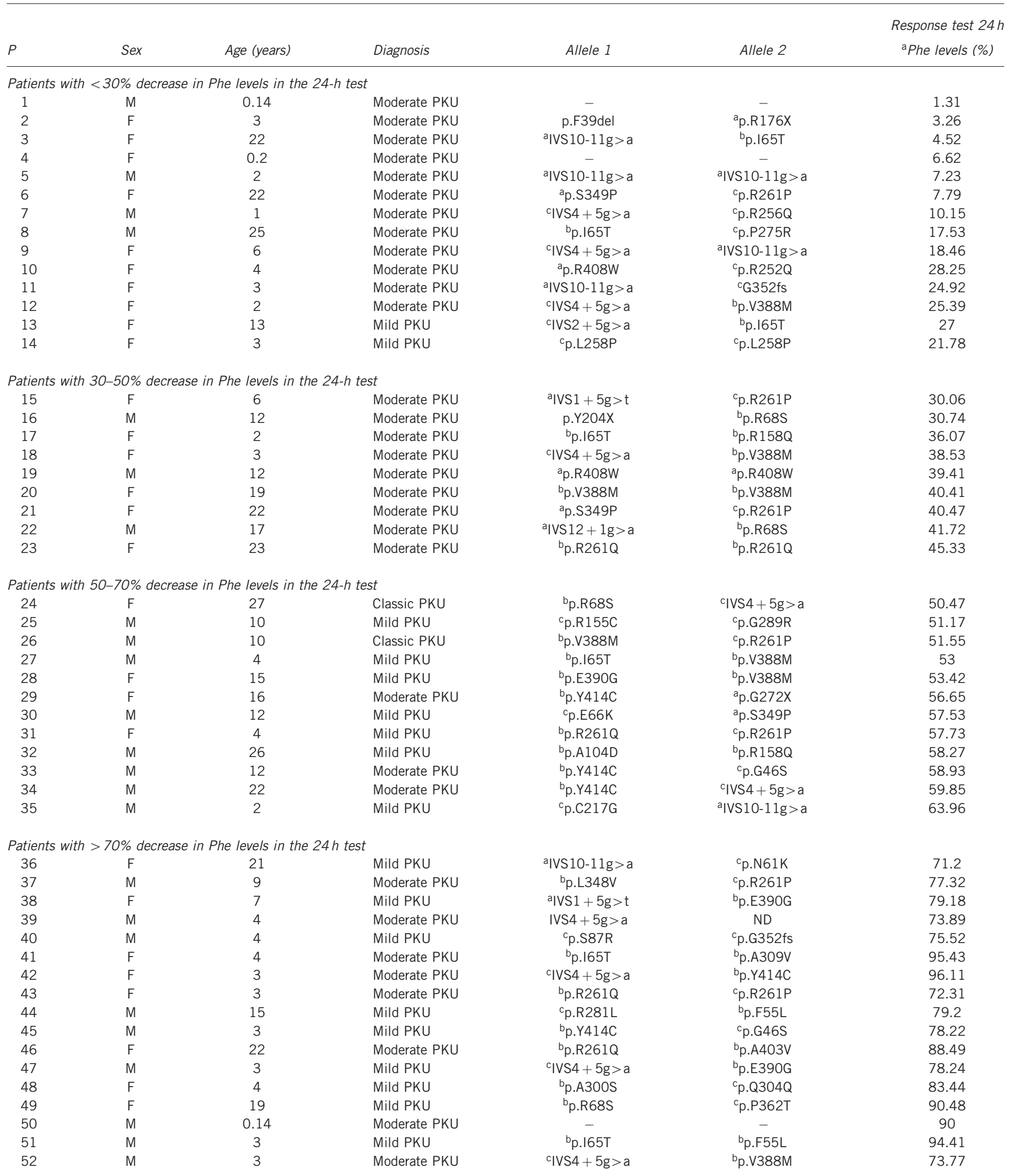

Abbreviations: F, female; M, male; ND, not determined; P, patient number; PKU, phenylketonuria Age at the time of the $\mathrm{BH} 4$ loading test.

Non-responsive classic PKU patients are not included.

a Mutations not associated with $\mathrm{BH}_{4}$ responsiveness.

${ }^{\mathrm{b}}$ Mutations previously associated with $\mathrm{BH}_{4}$ responsiveness.

${ }^{c}$ Mutations with yet undefined association to $\mathrm{BH}_{4}$ responsiveness. 
observed, there is no exact correlation for 8 of the 19 repeated genotypes (Table 2).

In 96 PKU subjects (18 mild PKUs, 32 moderate PKU, and 46 classic PKU) BH4 challenge was carried out. The test was positive in 29 patients and no response was found in 67 . Some of the responsive genotypes have been reported in a previous study aimed at evaluating the protocol for detecting $\mathrm{BH} 4$ responsiveness and the population to be tested. ${ }^{22}$ All patients with mutations previously associated with $\mathrm{BH}_{4}$ responsiveness in the two alleles had a clear positive response to the test, except p.20 with moderate PKU and homozygous for p.V388M, who exhibited $40.41 \%$ decrease in Phe levels $24 \mathrm{~h}$ after the challenge (Table 3 ).

Phenylketonuric patients who did not respond in the 24-h test, underwent a second stage of protocol for 1 week. This test was positive in two others patients (p22 and p23) who had a decrease phenylalanine levels between $40-50 \%$ with the 24 -h test.

\section{DISCUSSION}

We report the mutational spectrum of PAH deficiency in a cohort of 147 patients from Andalusia, exhibiting a high genetic heterogeneity, with 65 different mutations, most of them falling into the category of missense types. The results are in accordance with previous reports from South European patients, ${ }^{16,23-26}$ except for the Turkish population where there is a high rate of consanguinity, ${ }^{7}$ and are clearly different form North and central European countries, where the prevalent mutations are IVS $12+1 \mathrm{~g}>\mathrm{c}$ and p.R408W. ${ }^{14,27}$ Interestingly, the mutational spectrum in Andalusia is quite different from the one observed in the closely related Moroccan population, where the most frequent mutation was p.G352fsdelG found in $62.5 \%$ of the PKU alleles in an preliminary study. ${ }^{28}$ However, the second most frequent mutation after IVS10-11g $>\mathrm{a}$ in our population is p.S349P, which is particularly common in North African Jews with PKU. ${ }^{29}$

Four novel mutations were identified in five patients: p.L258P in two patients and p.E66K, p.R155C and p.P122S in one patient each. These mutations are associated with mild phenotypes and with a positive response to $\mathrm{BH} 4$; p.L258P is present in two mild PKU homozygous patients; p.E66K is in compound heterozygosity with the functionally null mutation p.S349P; p.R155C is combined with the severe mutation p.G289R also in mild PKU patients; and p.P122S is in compound heterozygosity with the mild mutation p.D415N. (In this case both alleles could be contributing to the mild phenotype and $\mathrm{BH} 4$ responsiveness, also taking into account the mutation p.P122Q affecting the same amino acid has been reported in some cases to be associated to a positive $\mathrm{BH} 4$ response (ref. BIOPKU)).

Overall, there is a good genotype-phenotype correlation taking into account previously reported associated phenotypes and residual activities of the mutant proteins, with patients carrying null mutations in both alleles showing the highest degree of concordance with the most severe phenotypes as previously reported. ${ }^{10}$ However, there are siblings with identical genotype exhibiting slightly different phenotypes (patients have been re-evaluated) according to the standard classification, confirming previous studies regarding interindividual phenotypic variability, which could be due to genetic and non-genetic factors affecting processes such as intestinal absorption, hepatic $\mathrm{BH} 4{ }^{20}$ and Phe metabolism or cellular protein quality control managing mutant $\mathrm{PAH}$ proteins. ${ }^{14,15,30}$

Regarding BH4 responsiveness, previous studies have shown that the genotype is the main determinant. The present study with patients from south Spain reveals that having on both alleles a mutation not associated to $\mathrm{BH} 4$ response is a reliable predictor of the absence of
BH4 responsiveness. On the other hand, $95.5 \%$ of the responsive patients carry at least one missense mutation previously associated to the $\mathrm{BH} 4$ response. All these patients have mild PKU. In addition, it is interesting to note that the rate of decrease in Phe levels and the lowest values achieved vary between patients with different genotypes but appears to be similar in patients with the same genotype. These data differ from the conclusions of some studies stating the difficulty in predicting accurately the degree of $\mathrm{BH} 4$ responsiveness based on the genotype, as patients with the same genotype exhibited different responses to the $\mathrm{BH} 4$ challenge. ${ }^{31,32}$ This inconsistency within the same genotype could be due to non-standardized BH4 loading test, in fact an important difference of these studies compared with ours is the variability in the $\mathrm{BH} 4$ loading test in the different centers included in the study. ${ }^{32}$ Increased protein breakdown due to a catabolic condition of the patient during the test could also influence the results.

In conclusion, a spectrum of 147 disease-causing mutations in Andalusian subjects with PAH deficiency was identified. Sixty-five different mutations including four novel ones were identified. A good correlation between the genotype and biochemical and BH4-responsive phenotype was observed. It is important to perform a standardized wellcontrolled BH4 load test in large cohort of well-studied patients to definitely assess $\mathrm{BH} 4$ responsiveness associated to each genotype.

1 Mathias, D. \& Bickel, H. Follow-up study of 16 years neonatal screening for inborn errors of metabolism in West Germany. Eur. J. Pediatr. 145, 310-312 (1986).

2 Delgado Pellecín, C., García-Valdecasas, M. S., Bueno Delgado, M. A., Pérez-Cerdá, C. \& Merinero Cortés, B. Experiencia del cribado neonatal ampliado en el Hospital Universitario Virgen del Rocío. Acta. Pediatr. Esp. 69, 89 (2011).

3 Mitchell, J. J., Trakadis, Y. J. \& Scriver, C. R. Phenylalanine hydroxylase deficiency. Genet. Med. 13, 697-707 (2011)

4 Kozak, L., Hrabincova, E., Kintr, J., Horky, O., Zapletalova, P., Blahakova, I. et al. Identification and characterization of large deletions in the phenylalanine hydroxylase (PAH) gene by MLPA: evidence for both homologous and non-homologous mechanisms of rearrangement. Mol. Genet. Metab. 89, 300-309 (2006).

5 Desviat, L. R., Pérez, B. \& Ugarte, M. Identification of exonic deletions in the PAH gene causing phenylketonuria by MLPA analysis. Clin. Chim. Acta. 373, 164-167 (2006).

6 Quirk, M. E., Dobrowolski, S. F., Nelson, B. E., Coffee, B. \& Singh, R. H. Utility of phenylalanine hydroxylase genotype for tetrahydrobiopterin responsiveness classification in patients with phenylketonuria. Mol. Genet. Metab. 107, 31-36 (2012).

7 Dobrowolski, S. F., Heintz, C., Miller, T., Ellingson, C., Ozer, I., Gökçay, G. et al. Molecular genetics and impact of residual in vitro phenylalanine hydroxylase activity on tetrahydrobiopterin responsiveness in Turkish PKU population. Mol. Genet. Metab. 102, 116-121 (2011).

8 Waters, P. J., Parniak, M. A., Nowacki, P. \& Scriver, C. R. In vitro expression analysis of mutations in phenylalanine hydroxilase: linking genotype to phenotype and structure to function. Hum. Mutat. 11, 4-17 (1998).

9 Dobrowolski, S. F., Borski, K., Ellingson, C. C., Koch, R., Levy, H. L. \& Naylor, E. W. A limited spectrum of phenylalanine hydroxylase mutations is observed in phenylketonuria patients in western Poland and implications for treatment with 6R tetrahydrobiopterin. J. Hum. Genet. 54, 335-339 (2009).

10 Santos, L. L., Fonseca, C. G., Starling, A. L., Januário, J. N., Aguiar, M. J., Peixoto, M. G. et al. Variations in genotype-phenotype correlations in phenylketonuria patients. Genet. Mol. Res. 9, 1-8 (2010).

11 Pey, A. L., Stricher, F., Serrano, L. \& Martinez, A. Predicted effects of missense mutations on native-state stability account for phenotypic outcome in phenylketonuria, a paradigm of misfolding diseases. Am. J. Hum. Genet. 81, 1006-1024 (2007).

12 Erlandsen, H., Pey, A. L., Gamez, A., Pérez, B., Desviat, L. R., Aguado, C. et al. Correction of kinetic and stability defects by the cofactor $\mathrm{BH} 4$ in $\mathrm{PKU}$ patients with certain phenylalanine hydroxylase mutations. Proc. Natl Acad. Sci. USA 101, 16903-16908 (2004).

13 Tyfield, L. A., Zschocke, J., Stephenson, A., Cockburn, F., Harvie, A., Bidwell, J. L. et al. Discordant phenylketonuria phenotypes in one family: the relationship between genotype and clinical outcome is a function of multiple effects. J. Med. Genet. 32, 867-870 (1995).

14 Guldberg, P., Levy, H. L., Koch, R., Berlin, C. M., Francois, B., Henriksen, K. F. et al. Mutation analysis in families with discordant phenotypes of phenylalanine hydroxylase deficiency: inheritance and expression of the hyperphenylalaninemia. J. Inherit. Metab. Dis. 17, 645 (1994).

15 Kayaalp, E., Treacy, E., Waters, P. J., Byck, S., Nowacki, P. \& Scriver, C. R. Human phenylalanine hydroxylase mutations and hyperphenylalaninemia phenotypes: a met analysis of genotype-phenotype correlations. Am. J. Hum. Genet. 61, 1309-1317 (1997). 
16 Zschocke, J. Phenylketonuria mutations in Europe. Hum. Mutat. 21, 345-356 (2003). 17 Desviat, L. R., Pérez, B., Gámez, A., Sánchez, A., García, M. J., Martínez-Pardo, M. et al. Genetic and phenotypic aspects of phenylalanine hydroxylase deficiency in Spain: molecular survey by regions. Eur. J. Hum. Genet. 7, 386-392 (1999).

18 Daniele, A., Cardillo, G., Pennino, C., Carbone, M. T., Scognamiglio, D., Correra, A. et al. Molecular epidemiology of phenylalanine hydroxylase deficiency in Southern Italy: a 96\% detection rate with ten novel mutations. Ann. Hum. Genet. 71, 185-193 (2007).

19 Zare-Karizi, S. H., Hosseini-Mazinani, S. M., Khazaei-Koohpar, Z., Seifati, S. M. Shahsavan-Behboodi, B., Akbari, M. T. et al. Mutation spectrum of phenylketonuria in Iranian population. Mol. Genet. Metab. 102, 29-32 (2011).

20 Desviat, L. R., Perez, B., García, M. J., Martínez-Pardo, M., Baldellou, A., Arena, J. et al. Relationship between mutation genotype and biochemical phenotype in a heterogeneous Spanish phenylketonuria population. Eur. J. Hum. Genet. 5, $196-$ 202 (1997).

21 Okano, Y., Kudo, S., Nishi, Y., Sakaguchi, T. \& Aso, K. Molecular characterization of phenylketonuria and tetrahydrobiopterin-responsive phenylalanine hydroxylase deficiency in Japan. J. Hum. Genet. 56, 306-312 (2011).

22 Bueno, M. A., Lage, S., Delgado, C., Andrade, F., Couce, M. L., González-Lamuño, D. et al. New evidence for assessing tetrahydrobiopterin $(\mathrm{BH}(4))$ responsiveness. Metabolism 61, 1809-1816 (2012).

23 Rivera, I., Mendes, D., Afonso, A., Barroso, M., Ramos, R., Janeiro, P. et al. Phenylalanine hydroxylase deficiency: molecular epidemiology and predictable $\mathrm{BH} 4$ responsiveness in South Portugal PKU patients. Mol. Genet. Metab. 104, S86-S92 (2011).

24 Pérez, B., Desviat, L. R. \& Ugarte, M. Analysis of the phenylalanine hydroxylase gene in the spanish population: mutation profile and association with intragenic polymorphic markers. Am. J. Hum. Genet. 60, 95-102 (1997).
25 Dianzani, I., Giannattasio, S., de Sanctis, L., Marra, E., Ponzone, A., Camaschella, C. et al. Genetic history of phenylketonuria mutations in Italy. Am. J. Hum. Genet. 55, 851-853 (1994).

26 Khemir, S., Tebib, N., Nasrallah, F., Ben Nour, F., Mizouni, H., Elasmi, M. et al. Phenylketonuria in Tunisian institutions for the mentally handicapped. Arch. Dis. Child 94, 647-648 (2009).

27 Sterl, E., Paul, K., Paschke, E., Zschocke, J., Brunner-Krainz, M., Windisch, E. et al. Prevalence of tetrahydrobiopterine (BH4)-responsive alleles among Austrian patients with PAH deficiency: comprehensive results from molecular analysis in 147 patients. J. Inherit. Metab. Dis. 36, 7-13 (2013).

28 Dahri, S., Desviat, L. R., Pérez, B., Leal, F., Ugarte, M. \& Chabraoui, L. Mutation analysis of phenylketonuria patients from Morocco: high prevalence of mutation G352fsdelG and detection of a novel mutation p.K85X. Clin. Biochem. 43, 76-81 (2010).

29 Weinstein, M., Eisensmith, R. C., Abadie, V., Avigad, S., Lyonnet, S., Schwartz, G. et al. A missense mutation, S349P, completely inactivates phenylalanine hydroxylase in North African Jews with phenylketonuria. Hum. Genet. 90, 645-649 (1993).

30 Guldberg, P., Rey, F., Zschocke, J., Romano, V., François, B., Michiels, L. et al. A European multicenter study of phenylalanine hydroxylase deficiency: classification of 105 mutations and a general system for genotype-based prediction of metabolic phenotype. Am. J. Hum. Genet. 63, 71-79 (1998).

31 Lindner, M., Haas, D., Mayatepek, E., Zschocke, J. \& Burgard, P. Tetrahydrobiopterin responsiveness in phenylketonuria differs between patients with the same genotype. Mol. Genet. Metab. 73, 104-106 (2001).

32 Trefz, F. K., Scheible, D., Götz, H. \& Frauendienst-Egger, G. Significance of genotype in tetrahydrobiopterin-responsive phenylketonuria. J. Inherit. Metab. Dis. 32, 22-26 (2009). 